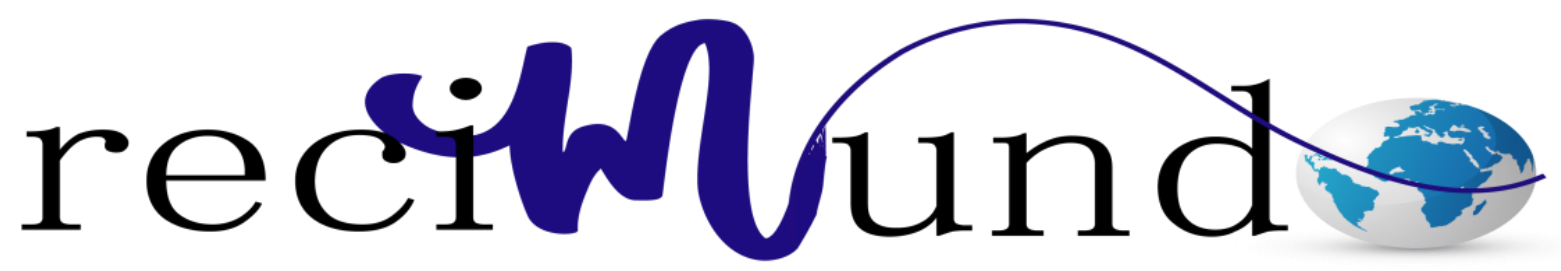

Revista Científica Mundo de la Investigación y el Conocimiento

Ana María León Pazmiño ${ }^{\text {a; }}$ Andrea Patricia Albán Vinueza ${ }^{\text {b }}$

Incidencia del trabajo colaborativo mediante la herramienta informática Google

Drive, en el desarrollo de la expresión escrita del idioma inglés

Incidence of collaborative work through the Google Drive computer tool, in the development of the written expression of the English language

Revista Científica Mundo de la Investigación y el Conocimiento. Vol. 2 núm.4,

Octubre, ISSN: 2588-073X, 2018, pp. 114-138

DOI: 10.26820/recimundo/2.(4).octubre.2018.114-138

URL: http://www.recimundo.com/index.php/es/article/view/342

Editorial Saberes del Conocimiento

Recibido: 15/07/2018 Aceptado: 05/08/2018Ｐublicado: 30/10/2018

Correspondencia: andydehernan@gmail.com

a. Lingüística Aplicada a la Enseñanza de Idiomas Especialidad Inglés; Ciencias de la Educación Mención: Investigación Educativa; Lingüística y Didáctica de la Enseñanza de Idiomas Extranjeros; Universidad Central del Ecuador.

b. Licenciatura en Ciencias de la Educación Especialidad Plurilingue; Maestría en Gerencia y Liderazgo Educacional; Universidad Central del Ecuador. 


\section{Incidencia del trabajo colaborativo mediante la herramienta informática Google Drive, en el desarrollo de la expresión escrita del idioma inglés}

Vol. 2, núm. 4., (2018)

Ana María León Pazmiño; Andrea Patricia Albán Vinueza

\section{RESUMEN}

En el presente artículo abordó la importancia del trabajo colaborativo en el desarrollo de las habilidades de la expresión escrita en inglés como una metodología que logra mejorar los procesos de enseñanza aprendizaje en las aulas universitarias. El diseño de la investigación fue de tipo cualitativa, correlacional, cuasi experimental con el cual se pudo confirmar un cierto grado de importancia que tiene la implementación de trabajos colaborativos, mediante la implementación de la herramienta Google Drive, para mejorar la habilidad de escritura en los y las alumnos, concluyendo que este proceso permite identificar actividades adecuadas para mejorar la habilidad de la expresión escrita.

Palabras clave: Ingles, informática, google drive, trabajo colaborativo, escritura. 


\title{
Incidencia del trabajo colaborativo mediante la herramienta informática Google Drive, en el desarrollo de la expresión escrita del idioma inglés
}

Vol. 2, núm. 4., (2018)

Ana María León Pazmiño; Andrea Patricia Albán Vinueza

\begin{abstract}
This article addressed the importance of collaborative work in the development of the skills of the expression written in English as a methodology that manages to improve the processes of teaching and learning in the University classroom. The research design was of the type qualitative, correlational, quasi-experimental with which it was able to confirm a certain degree of importance which is the implementation of collaborative work, through the implementation of the Google Drive tool, to improve the ability of writing in the students, concluding that this process allows to identify appropriate activities to improve the ability of writing.
\end{abstract}

Keywords: English, computer science, google drive, collaborative work, writing. 


\section{Incidencia del trabajo colaborativo mediante la herramienta informática Google Drive, en el desarrollo de la expresión escrita del idioma inglés}

Vol. 2, núm. 4., (2018)

Ana María León Pazmiño; Andrea Patricia Albán Vinueza

\section{Introducción.}

La puesta en escena sobre la importancia del conocimiento del idioma inglés en el ámbito profesional, ha demandado la necesidad de plantear una serie de estrategias tendientes a su dominio como segunda lengua; por lo que uno de los retos más grandes que cada institución educativa tiene es la de lograr que sus educandos lleguen a tener una suficiencia en los que se refiere a las cuatro habilidades habla, escucha, lectura y escritura; donde el desarrollo de la expresión escrita, dentro del proceso de enseñanza-aprendizaje, es uno de los objetivos fundamentales para la adquisición de una segunda lengua.

Desafortunadamente, los estudiantes presentan serias dificultades en plasmar sus ideas donde se les hace muy complicado, inclusive, elaborar una oración sencilla; por lo que es imprescindible que los docentes utilicen metodologías y estrategias adecuadas a las necesidades de sus estudiantes.

En los últimos años se ha evidenciado que el aprendizaje de la expresión escrita mejora notablemente si se hace uso de herramientas tecnológicas como es el caso de la herramienta Google Drive.

De este modo, la investigación se enfocó, por un lado, en la dificultad que presentan los estudiantes en el aprendizaje de la expresión escrita en niveles básicos del idioma Inglés de la Universidad Central del Ecuador, de la ciudad de Quito y, por otro, planteó como herramienta del trabajo colaborativo el uso de la herramienta tecnológica de Google Drive. 


\section{Incidencia del trabajo colaborativo mediante la herramienta informática Google Drive, en el desarrollo de la expresión escrita del idioma inglés}

Vol. 2, núm. 4., (2018)

Ana María León Pazmiño; Andrea Patricia Albán Vinueza

Se entiende así la trascendencia de la investigación para el desarrollo de la educación actual en países de la región como Ecuador, ya que la escasa utilización de herramientas tecnológicas al momento de enseñar un segundo idioma no promueve una mejor producción escrita del inglés como lengua extranjera en los estudiantes. Por esta razón, el planteamiento, ejecución y resultados de esta investigación ayudarán a mejorar de forma didáctica el proceso enseñanza-aprendizaje del inglés como lengua extranjera.

Es necesario para entender la importancia del tema que se aborda en este artículo ir definiendo los conceptos clave que motivan la investigación, conceptos como aprendizaje colaborativo, Google drive, El aprendizaje colaborativo a través de Google Drive

Aprendizaje colaborativo encierra muchas características que han sido definidos por varios autores, entre esos Echazarreta, Prados, Poch y Soler (2009) quienes entienden que el trabajo en grupo significa:

a) Una fuerte relación de interdependencia de los diferentes miembros que lo conforman, de manera que el alcance final de las metas concierna a todos los miembros;

b) Una clara responsabilidad individual de cada miembro del grupo para el alcance de la meta final;

c) La formación de los grupos en el trabajo colaborativo es heterogénea en habilidad, características de los miembros en oposición, en el aprendizaje tradicional de grupos, estos son más homogéneos; 


\section{Incidencia del trabajo colaborativo mediante la herramienta informática Google Drive, en el desarrollo de la expresión escrita del idioma inglés}

Vol. 2, núm. 4., (2018)

Ana María León Pazmiño; Andrea Patricia Albán Vinueza

d) Todos los miembros tienen su parte de responsabilidad para la ejecución

de las acciones en el grupo;

e) La responsabilidad de cada miembro del grupo es compartida y

f) Se persigue el logro de objetivos a través de la realización (individual y conjunta) de tareas (pág. 29).

Es una tarea que implica sobre todo el compromiso de cada miembro del grupo para conseguir los objetivos. Su éxito reside principalmente en la planificación de las actividades por parte del docente en el que se deba delegar tareas que promueva la participación de todos los y las estudiantes, o a su vez que aporten en el desarrollo de la organización de los contenidos.

Pero es importante también que dentro del trabajo colaborativo exista reciprocidad que lo señala como el "sistema de prestaciones mutuas que se dan entre dos personas, dos grupos, dos poblaciones, etc. Estas prestaciones son intercambios simétricos de bienes y servicios, fundamentales para cada una de las partes involucradas" (Gonnet, 2010, pág. 2) y que en esencia señala que cada individuo participe durante todo el proceso y desarrollo de la actividad, cuya base es el respeto por cada función otorgada para facilitar la ejecución de la tarea. Todos deben ser capaces de argumentar sus respuestas, es decir, en desarrollar el pensamiento crítico que contribuya a la resolución de conflictos o situaciones que el equipo deba responder adecuadamente.

Woolfolk (1999) citado por Maldonado (2007), explica que "los componentes del trabajo colaborativo funcionan de acuerdo a un objetivo en común, en la interdependencia de metas, de 


\section{Incidencia del trabajo colaborativo mediante la herramienta informática Google Drive, en el desarrollo de la expresión escrita del idioma inglés}

Vol. 2, núm. 4., (2018)

Ana María León Pazmiño; Andrea Patricia Albán Vinueza

recursos, de roles y de premios" (pág. 269). Sin embargo, hay que tener en cuenta que todas estas

características se relacionan unas con otras y depende del grado de compromiso individual como de la organización del grupo.

Google drive Es una herramienta útil para el manejo de información. Se define como:

"Un servicio web que te permite almacenar, modificar, compartir y acceder a sus archivos y documentos independientemente de dónde te encuentres a través de internet. Puedes subir al servicio más de 30 tipos de archivos entre los que se incluyen vídeos en alta definición, PSD de Photoshop® o AI de Adobe Ilustrator®” (Ibilib, 2013, pág. 7)

El aprendizaje colaborativo a través de Google Drive en el aula o fuera de ella, Google Drive es coherente con los objetivos de la pedagogía constructivista, pues permite realizar tareas en pequeños grupos de manera colaborativa y cooperativa las 24 horas del día, dejando a un lado el tradicional papel y lápiz para poder comunicarse correctamente de manera escrita entendiéndose que los y las estudiantes tienen la opción de convertir el aula tradicional en un espacio de construcción de su conocimiento, a través de la experiencia vivida desde su aula virtual y durante la interacción con los otros miembros del grupo, favoreciendo tanto a su desarrollo socio-afectivo como al desarrollo de sus habilidades de pensamiento en la construcción de párrafos coherentes y cohesivos.

La propuesta de realizar un trabajo colaborativo mediante la herramienta informática Google Drive, en el desarrollo de la expresión escrita del idioma Inglés, puede ser aplicada en cualquier universidad, a través del diseño de una guía metodológica que permita a los y las estudiantes desarrollar habilidades para construir párrafos cohesivos y coherentes; permitiendo a 


\section{Incidencia del trabajo colaborativo mediante la herramienta informática Google Drive, en el desarrollo de la expresión escrita del idioma inglés}

Vol. 2, núm. 4., (2018)

Ana María León Pazmiño; Andrea Patricia Albán Vinueza

los y las maestras trabajar en colaboración con sus alumnos, otorgándoles una respuesta inmediata en tiempo real.

Se entiende así que los alumnos le dan más importancia al significado que a la forma donde "este tipo de tecnologías modernas contribuye a que los aprendientes desarrollen un sentido de pertenencia hacia el texto que construyen en conjunto, desarrollando, a la vez, un cierto grado de autonomía respecto del profesor (Ubilla, Gómez, \& Sáez, 2017, pág. 333); con lo que se destaca que estos recursos ya han empezado a utilizarse en el aula donde se ha evidenciado que se generan espacios de comunicación propicios para el desarrollo de habilidades comunicativas como la producción de la escritura en otro idioma. Así lo señalan Ubilla, Gómez y Sáez (2017) quienes destacan que esta herramienta forja "actitudes de un nuevo tipo de alfabetización tecnológica crítica, colaborativa y creativa. Del mismo modo, estas herramientas permiten crear un entorno práctico eficiente para la socialización y la culturización de aprendientes (pág. 334).

Se evidencia así que para el desarrollo de la competencia de la producción de la escritura en inglés es válido el uso de herramientas tecnológicas como Google Drive que se muestran a modo de trabajo colaborativo que facilita y fortalece el aprendizaje de otro idioma mediante un sistema mucho más dinámico y participativo

Esta herramienta de acceso gratuito fue creado precisamente para la educación básica y universitaria donde ni siquiera requiere de instalación de un software alguno, por lo que su aprovechamiento en el aula es significativo sobre todo si lo que se desea es desarrollar competencias en la escritura de un modo didáctico y participativo; donde los estudiantes se 


\section{Incidencia del trabajo colaborativo mediante la herramienta informática Google Drive, en el desarrollo de la expresión escrita del idioma inglés}

Vol. 2, núm. 4., (2018)

Ana María León Pazmiño; Andrea Patricia Albán Vinueza

convierten en responsables de su propio aprendizaje; así como también un apoyo en el aprendizaje de sus compañeros; quienes intercambiarán criterios e ideas; promoviendo así el desarrollo del pensamiento crítico de los participantes

\section{Metodología.}

El diseño de la investigación fue de tipo cualitativa, correlacional, cuasi experimental; en virtud de que no se cumplieron a cabalidad todas las características que, por definición, acompañan al método de investigación experimental, ya que, en este estudio, no se realizó la selección estadísticamente aleatoria de los participantes de la muestra, sino que la selección se la hizo de forma intencional (Hernández, 2010, pág. 188).

La presente investigación contó así con el grupo control y el grupo experimental. A los dos grupos se les administró un pre-test y un post-test. Cabe destacar, que con el grupo control, se trabajó con una metodología tradicional; mientras que con el grupo experimental, se trabajó con una metodología colaborativa a través de la herramienta informática de Google Drive.

La población objeto de estudio, correspondió a estudiantes de la Universidad Central del Ecuador, de nivel básico con un nivel del idioma inglés básico. Se trabajará con dos grupos, uno de control y otro experimental. Cada grupo o muestra estuvo compuesta por 30 estudiantes, hombres y mujeres de diferentes carreras, con un nivel de inglés básico; dando un total de 60 estudiantes. La muestra escogida hace referencia al número promedio de estudiantes existentes en cada aula. 


\section{Incidencia del trabajo colaborativo mediante la herramienta informática Google Drive, en el desarrollo de la expresión escrita del idioma inglés}

Vol. 2, núm. 4., (2018)

Ana María León Pazmiño; Andrea Patricia Albán Vinueza

Esta investigación se la llevó a cabo durante cuatro semanas. En el grupo control se designaron cuatro horas presenciales, semanales, dando un total de 20 horas; dichas sesiones fueron impartidas dentro del horario regular de clases de 7 h00 a 9h00. Durante estas cuatro horas semanales, se trabajó en el desarrollo de la habilidad escrita a través del uso de una metodología tradicional.

Con lo que respecta al grupo experimental, las tutorías se las impartió fuera del horario regular de clases en vista de que el Centro de Idiomas no cuenta con laboratorios ni Internet; por lo tanto, las veinte sesiones se realizaron los días miércoles, sábados y domingos con un horario de 18 h00 a 20h00, utilizando una metodología colaborativa, a través de la herramienta Google Drive. Los 30 estudiantes fueron divididos en cinco grupos de seis respectivamente

En este tipo de investigación se analizaron las relaciones existentes entre la variable independiente: El trabajo colaborativo a través de la herramienta informática Google Drive"; y, la variable dependiente: "La expresión escrita y los efectos causales de las primeras sobre las segundas.

Cabe señalar, que esta investigación fue de tipo longitudinal pues el seguimiento de la investigación se la realizó con dos grupos de estudiantes, a través de un tiempo determinado, con el fin de analizar procesos de cambio.

\section{Resultados.}

Para el análisis e interpretación de resultados se tomó en cuenta los siguientes parámetros: proceso de escritura, ortografía, puntuación, gramática, estructura (título, idea 


\section{Incidencia del trabajo colaborativo mediante la herramienta informática}

Google Drive, en el desarrollo de la expresión escrita del idioma inglés

Vol. 2, núm. 4., (2018)

Ana María León Pazmiño; Andrea Patricia Albán Vinueza

principal, idea secundaria, y conclusión), secuencia y coherencia, contenido del párrafo. A continuación se exponen los resultados obtenidos:

\section{Gráfico 3. Proceso de escritura}

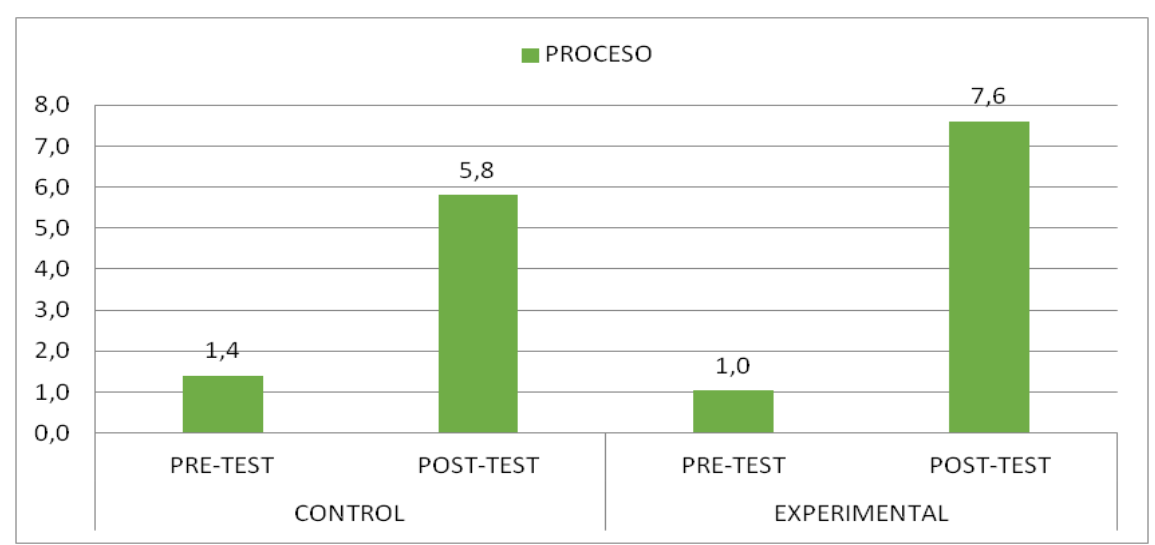

Elaborado por: Ana María León

Fuente: Investigación de campo.

En cuanto al proceso de escritura, se puede observar que tanto el grupo control como el experimental tuvieron un incremento en su calificación final; sin embargo, el grupo experimental presentó un incremento significativo con una diferencia de 6.6 puntos con respecto a la pre prueba; mientras que el grupo control incrementó en 4.2 puntos. 


\section{Incidencia del trabajo colaborativo mediante la herramienta informática}

Google Drive, en el desarrollo de la expresión escrita del idioma inglés

Vol. 2, núm. 4., (2018)

Ana María León Pazmiño; Andrea Patricia Albán Vinueza

\section{Gráfico 4. Proceso de ortografía}

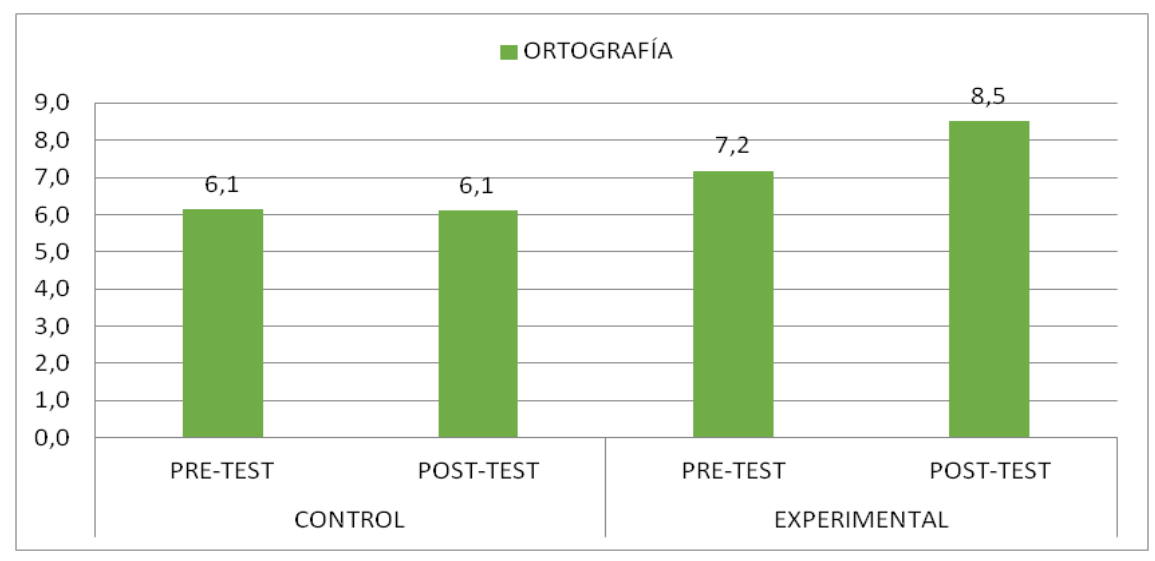

Elaborado por: Ana María León

Fuente: Investigación de campo.

En lo concerniente a la ortografía, el grupo control mantuvo una calificación similar tanto en la pre como en la post prueba. Esto nos lleva a la conclusión de que, con el uso del método tradicional, no se logra que los estudiantes mejoren su ortografía. Con respecto al grupo experimental, se puede evidenciar que la ortografía sí mejoró a través del trabajo colaborativo y su interacción y retroalimentación recibida por parte de sus pares y su profesora guía. 
Incidencia del trabajo colaborativo mediante la herramienta informática Google Drive, en el desarrollo de la expresión escrita del idioma inglés Vol. 2, núm. 4., (2018)

Ana María León Pazmiño; Andrea Patricia Albán Vinueza

\section{Gráfico 5. Puntuación}

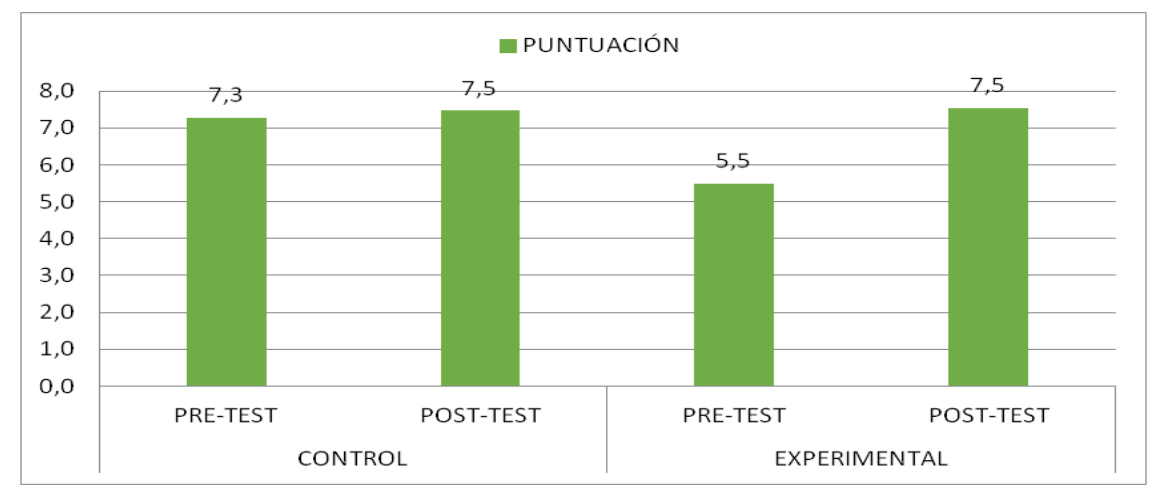

Elaborado por: Ana María León

Fuente: Investigación de campo.

Como se puede evidenciar, las calificaciones de la pre y post pueba son casi similares en el grupo control; mientras que el grupo experimental demuestra un incremento de dos puntos, ratificandose que el uso del trabajo collaborativo a través de la plataforma Google Drive sí incide en el uso correcto de puntuación. 


\section{Incidencia del trabajo colaborativo mediante la herramienta informática Google Drive, en el desarrollo de la expresión escrita del idioma inglés}

Vol. 2, núm. 4., (2018)

Ana María León Pazmiño; Andrea Patricia Albán Vinueza

\section{Gráfico 6. Gramática}

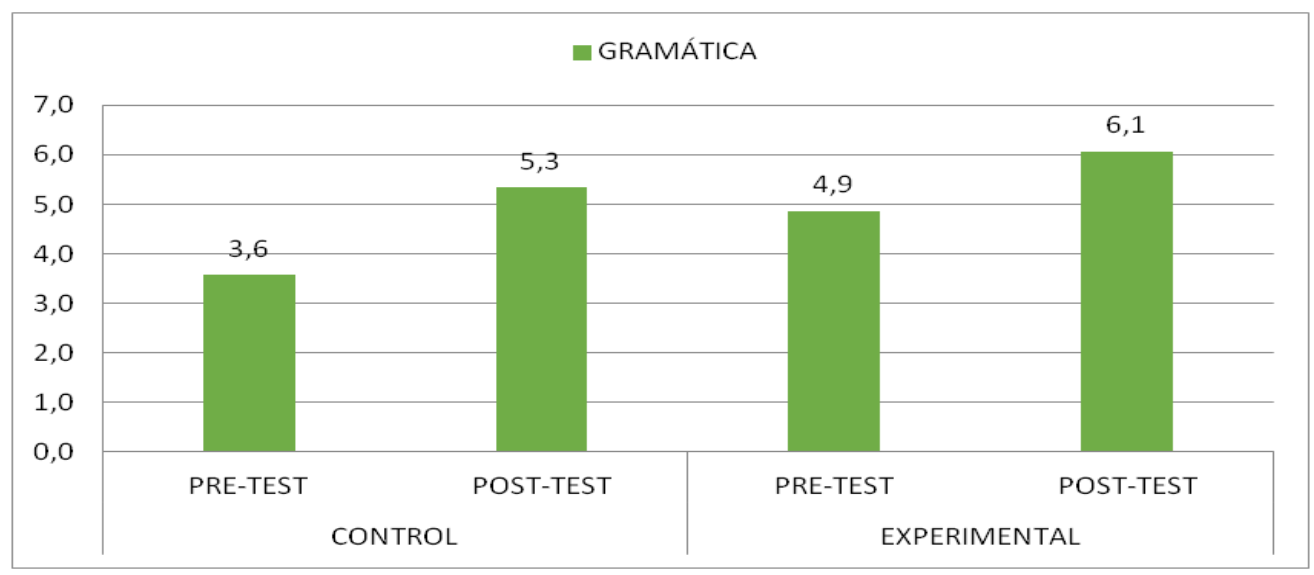

Elaborado por: Ana María León

Fuente: Investigación de campo.

Se puede observar que los resultados tanto del grupo control como experimental incrementaron su nota en lo que se refiere a la gramática. Esto permite evidenciar que el método tradicional con el que se trabajó con el grupo control y el método colaborativo utilizado en el grupo experimental, ayudaron a que los educandos utilicen correctamente las reglas gramaticales objeto de estudio. 
Incidencia del trabajo colaborativo mediante la herramienta informática Google Drive, en el desarrollo de la expresión escrita del idioma inglés Vol. 2, núm. 4., (2018)

Ana María León Pazmiño; Andrea Patricia Albán Vinueza

\section{Gráfico 7.Estructura}

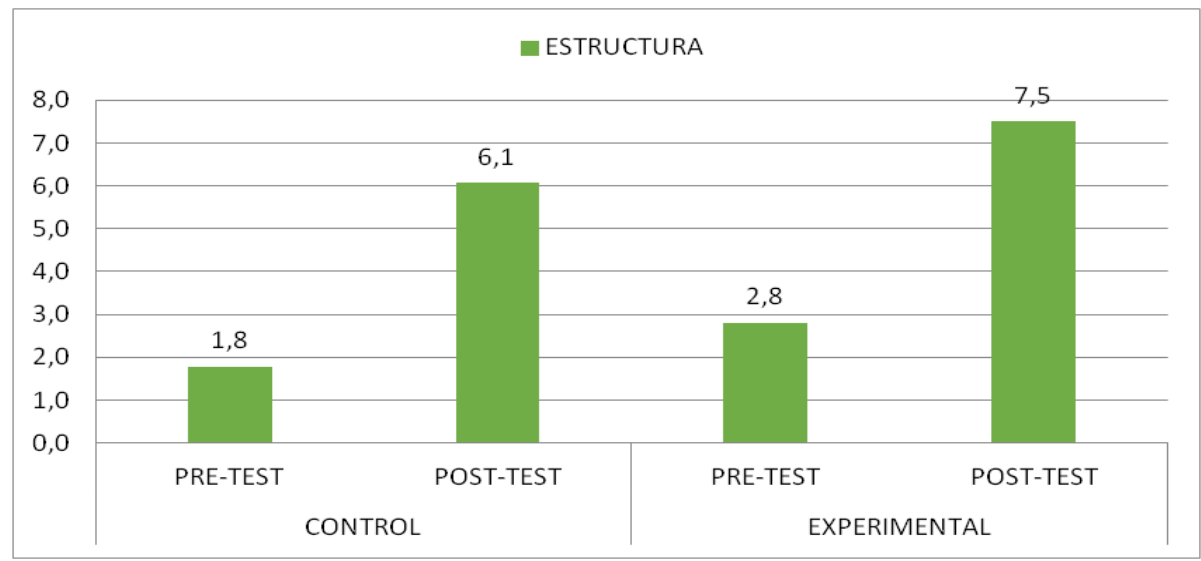

Elaborado por: Ana María León

Fuente: Investigación de campo.

En cuanto a la estructura (título, idea principal, idea secundaria, y conclusión) se evidencia un alto incremento en la calificación obtenida en ambos grupos; pero es mayor la significancia en el grupo experimental con una diferencia de 4.7 puntos en relación a su pre prueba. 


\section{Incidencia del trabajo colaborativo mediante la herramienta informática}

Google Drive, en el desarrollo de la expresión escrita del idioma inglés

Vol. 2, núm. 4., (2018)

Ana María León Pazmiño; Andrea Patricia Albán Vinueza

\section{Gráfico 8. Secuencia y Coherencia}

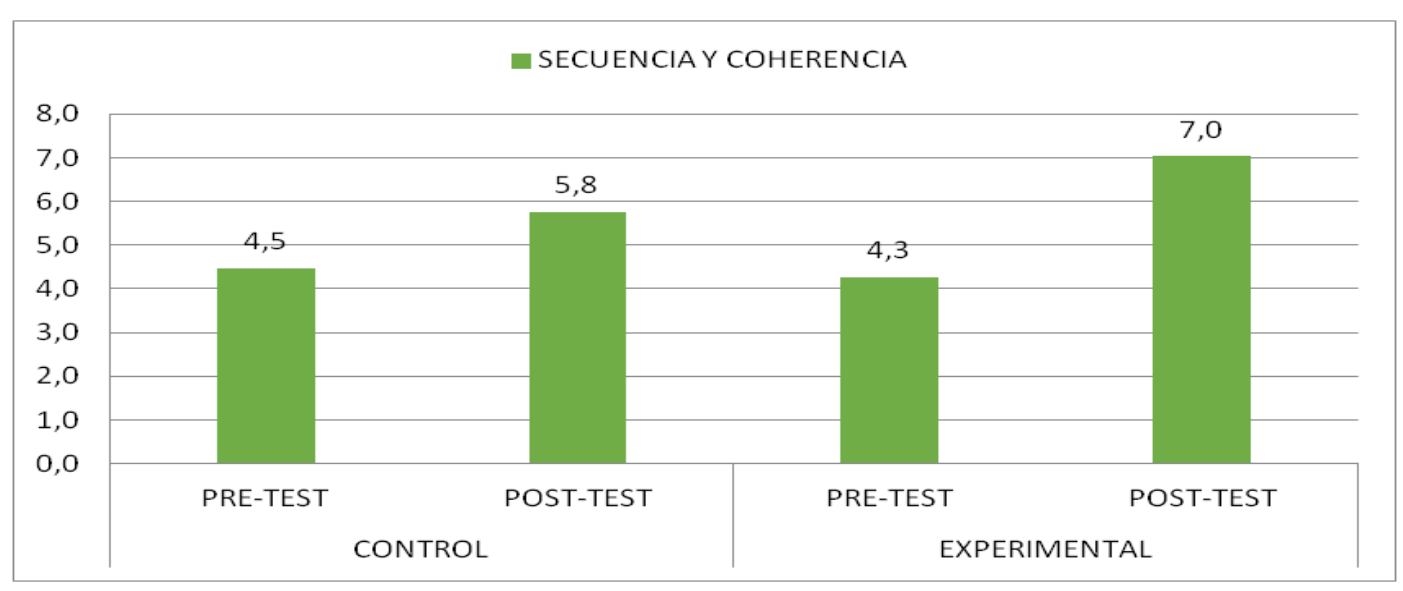

Elaborado por: Ana María León

Fuente: Investigación de campo.

Tanto el grupo control como el grupo experimental incrementaron su nota; sin embargo, los resultados de la post prueba del grupo experimento presenta un incremento de 2.7 puntos en relación con 1.30 correspondiente a la calificación obtenida en la post prueba del grupo control. 
Incidencia del trabajo colaborativo mediante la herramienta informática Google Drive, en el desarrollo de la expresión escrita del idioma inglés

Vol. 2, núm. 4., (2018)

Ana María León Pazmiño; Andrea Patricia Albán Vinueza

\section{Gráfico 1. Comparación}

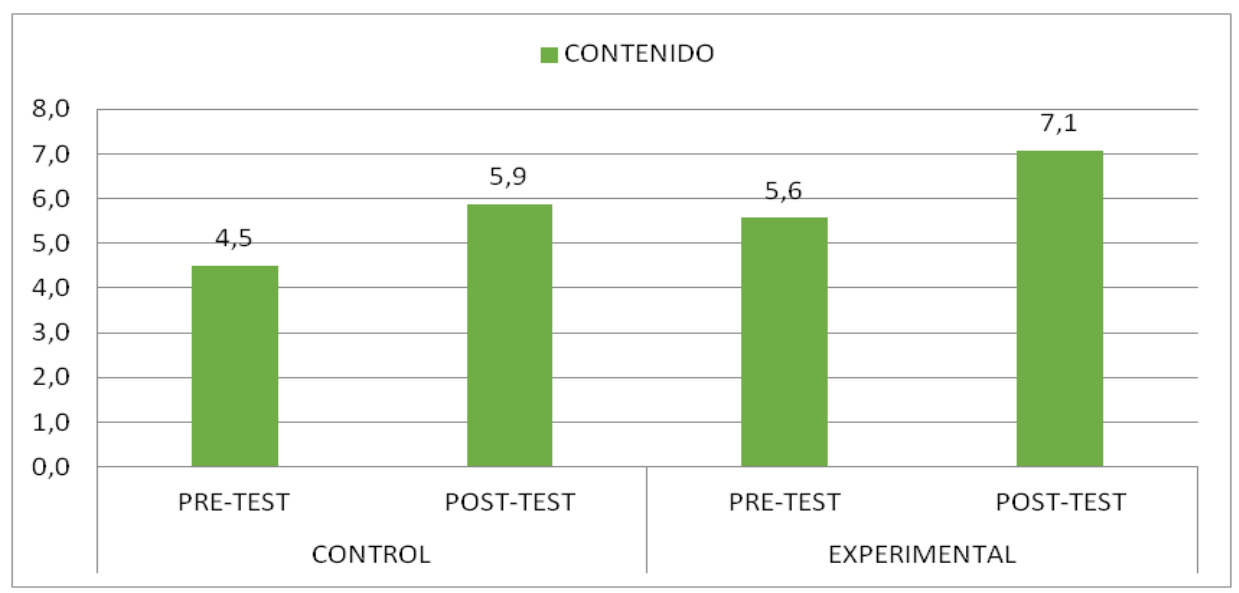

Elaborado por: Ana María León

Fuente: Investigación de campo.

Finalmente, en lo que respecta al contenido del párrafo, se evidencia un aumento de 1.4 con relación a la pre y post prueba del grupo control; y, 1.5 puntos de diferencia en lo que respecta a la pre y post prueba del grupo experimento. Lo que conlleva a decir que el método colaborativo sí influyó en el mejoramiento del contenido de los párrafos.

\section{Discusión.}

Haciendo una comparación del promedio de la nota obtenida en ambos grupos- antes y después del examen, se determinó que el promedio de notas inicial (Pre-test) en ambos grupos es bastante similar, con 4,16 puntos en el Grupo Control y 4,46 puntos en el Grupo Experimental. Lo que conlleva a la reflexión de que ambos grupos se encontraban inicialmente en situaciones 


\section{Incidencia del trabajo colaborativo mediante la herramienta informática Google Drive, en el desarrollo de la expresión escrita del idioma inglés}

Vol. 2, núm. 4., (2018)

Ana María León Pazmiño; Andrea Patricia Albán Vinueza

similares, pues sus conocimientos previos sobre la estructuración y elaboración de un párrafo eran desconocidos en la mayoría de los observados.

Sin embargo, al evaluarse por segunda vez a ambos grupos, y luego de haber sido impartidas las clases de forma colaborativa con el grupo experimental, se puede observar el incremento de 2,88 puntos en relación con el puntaje obtenido en su pre prueba; mientas que el grupo control experimentó un incremento de 1,98 en relación con su pre prueba.

Como se puede observar, los dos grupos, tanto el control como el experimental, incrementaron su calificación post-prueba, en comparación con la obtenida en la pre prueba. Sin embargo, el grupo experimental presentó un incrementó significativamente mayor en cuanto a su calificación media, puesto que la misma se incrementó un 64,7\%, en contraste el grupo control, en cuyo caso su calificación promedio aumentó en un 45,7\% durante los dos periodos evaluados (antes y después).

Posteriormente, se establecerá una prueba de hipótesis para determinar si es significativo - a un nivel de error del 5\%- el resultado obtenido. Y de esta manera corroborar la hipótesis alterna de que el utilizar la metodología propuesta sí influye notablemente en el rendimiento y aprendizaje de los y las estudiantes.

En lo que respecta a la comparación de la desviación estándar de las notas, obtenidas en ambos grupos antes y después del examen se pudo apreciar que se puede apreciar, en el grupo control se redujo la desviación estándar en 54.8\%, puesto que inicialmente presentó una desviación estándar de sus notas de examen previas a las clases (pre-test) de 0,93. Mientras que en la segunda evaluación, las notas del examen posterior a las sesiones (post-test) presentaron 
Incidencia del trabajo colaborativo mediante la herramienta informática Google Drive, en el desarrollo de la expresión escrita del idioma inglés

Vol. 2, núm. 4., (2018)

Ana María León Pazmiño; Andrea Patricia Albán Vinueza

una desviación estándar de 0.42. De igual manera, el grupo experimental disminuyó su desviación estándar en $15.52 \%$, puesto que en el pre-test su desviación era de 1,16 y en el posttest se presentó una desviación de 0.98 .

Y, finalmente, en comparación a la varianza de las notas, obtenidas en ambos grupos antes y después del examen, se puede corroborar lo anteriormente descrito en la dispersión de los datos, observando la varianza de las calificaciones pre prueba y post prueba, donde en el grupo de control la varianza disminuyó $79.15 \%$, pasando de 0,86 a 0.18 . Por otro lado, la varianza en el grupo experimental disminuyó en un $28.15 \%$, de 1.35 a 0.97 .

Como se puede ver, tanto la varianza como la desviación estándar son medidas de variabilidad de los datos; es decir, que representan la distancia o la separación que tienen los datos frente a la media. La varianza se define como un promedio de las diferencia entre los datos con la media y ello elevado al cuadrado. La desviación estándar se define como la raíz cuadrada de la varianza. Por lo tanto, al reducirse ésta medida de dispersión significa que los datos están más cercanos a la media.

En este caso, se puede inferir que en el grupo control los alumnos tienden en mayor medida hacia la media del curso de 6.06. En el grupo experimental también se redujo la separación de los datos, aunque en menor medida que en el grupo control. Esto puede deberse a que todavía existen datos atípicos, con un mayor número de alumnos que sobrepasan la media del curso de 7.34. Esto puede observarse en los anexos 13 y 14 en los cuales se presentas calificaciones cada alumno tanto del grupo control como experimental. Si se analizan el grupo 


\section{Incidencia del trabajo colaborativo mediante la herramienta informática Google Drive, en el desarrollo de la expresión escrita del idioma inglés}

Vol. 2, núm. 4., (2018)

Ana María León Pazmiño; Andrea Patricia Albán Vinueza

control, apenas el $36.6 \%$ de los alumnos supera la media de 6.06 puntos; de hecho, todos los alumnos obtuvieron en su post-prueba una nota dentro del rango de 5.29 a 6.86 puntos.

Por otro lado, en el grupo control la disminución de la dispersión de los datos fue menor que en el otro grupo. Se puede analizar que el 43,3\% de los alumnos supera la media del curso de 7.34 puntos. En este grupo, el rango de calificaciones es más amplio que en el otro grupo, ya que todos los alumnos obtuvieron una nota final en su post-prueba que se encuentra dentro del rango de 5.71 a 9.14 puntos.

Esto pone en relieve un cierto grado de importancia que tiene la implementación de trabajos colaborativos, mediante la implementación de la herramienta Google Drive, para mejorar la habilidad de escritura en los y las alumnos; pues como se puede examinar, no solamente se incrementa el promedio del curso, sino que también se incrementa el rango de notas, permitiendo a los y las estudiantes mejorar sus conocimientos de manera amplia.

\section{Conclusiones.}

Perspectivas tempranas sobre el trabajo colaborativo a través de herramientas tecnológicas, han demostrado ser de gran beneficio, pero sobre todo de gran motivación para los y las estudiantes quienes utilizan internet, específicamente Google Drive, con el fin de comunicar sus pensamientos de manera escrita; pues proporciona a los estudiantes un medio menos amenazante para lograr este fin.

Así se demostró en el proceso investigativo llevado a cabo con 30 estudiantes de la Universidad Central del Ecuador quienes mantenían un nivel medio del conocimiento del idioma 
Incidencia del trabajo colaborativo mediante la herramienta informática Google Drive, en el desarrollo de la expresión escrita del idioma inglés

Vol. 2, núm. 4., (2018)

Ana María León Pazmiño; Andrea Patricia Albán Vinueza

inglés, desde donde a partir de la aplicación de un post-test (rúbrica) se pudo evidenciar que el trabajo colaborativo en función de los recursos de la web facilita la creación de comunidades virtuales y el trabajo en grupo para incentivar el diálogo y la participación activa de las y los estudiantes en el desarrollo de sus habilidades de la expresión escrita.

El trabajo significó un análisis teórico de la variable independiente trabajo colaborativo y de la variable dependiente expresión escrita. La primera se definió como un modelo de aprendizaje que utiliza una metodología basada en el modelo pedagógico de Vygotsky, donde el estudiante es el protagonista de los procesos de enseñanza aprendizaje. Promueve el trabajo interdisciplinario y el conocimiento compartido mediante el uso de herramientas digitales.

La segunda variable se definió dentro de la enseñanza de una segunda lengua, en este caso el inglés, como un sistema complejo de la comunicación humana que consiste en el proceso de construcción de mensajes escritos mediante el uso de competencias comunicativas y lingüísticas para elaborar textos con sentido, coherencia, cohesión y adecuación.

Los resultados evidenciaron así que el trabajo colaborativo es un modelo de aprendizaje interactivo que trabaja con herramientas digitales como el Google Drive para fomentar la reciprocidad, la responsabilidad, las relaciones afectivas, la interdependencia positiva y el trabajo compartido en los estudiantes del nivel de usuario básico del idioma inglés de la Universidad Central del Ecuador.

Esta investigación cuasi-experimental y correlacional diseñó un pre-test y un post-test mediante rúbricas que fueron aplicadas a un grupo de control y experimental de estudiantes universitarios de diferentes carreras del nivel básico del idioma inglés de la Universidad Central 


\section{Incidencia del trabajo colaborativo mediante la herramienta informática Google Drive, en el desarrollo de la expresión escrita del idioma inglés}

Vol. 2, núm. 4., (2018)

Ana María León Pazmiño; Andrea Patricia Albán Vinueza

del Ecuador para evaluar la expresión escrita. Los resultados de la rúbrica señalaron que existían deficiencias en la expresión escrita de ambos grupos. Luego de la aplicación de la metodología colaborativa, y a través de la post prueba, se evidencia un mejoramiento en la elaboración de los párrafos.

De este modo se plantea la necesidad de utilizar en el aula recursos didácticos desde la Internet donde herramientas como Google Drive facilitan el proceso de enseñanza-aprendizaje de otro idioma y que en el desarrollo de la competencia de producción de la escritura otorga resultados muy positivos; por lo que quienes prefieren expresarse de manera estricta deben fortalecer destrezas con el uso de herramientas necesarias para lograr expresar sus ideas.

Pero existe una dificultad en el proceso de enseñanza-aprendizaje entendiéndose que el docente no utiliza metodologías quien incluso faciliten el proceso de calificación entendiéndose que el educando no recibe retroalimentación de su profesor o profesora, pues él o ella deben corregir 30 párrafos como un promedio de estudiantes por aula; convirtiéndose este trabajo en algo desgastante para él o la maestra pues debe dedicar largo tiempo en las corrección individual de los trabajos; y lo que es peor aún la retroalimentación no es directa ni inmediata como se menciona anteriormente.

Se menciona, así mismo, que es imprescindible que el maestro o la maestra planifique su clase en base a las necesidades de sus estudiantes y no se rija tan solo a los temas que existen en la sección de escritura del libro que la institución siga; sino que provea a sus discentes de temas relacionados con sus vidas y su entorno con el fin de convertir a este proceso de aprendizaje un momento de esparcimiento. 
Incidencia del trabajo colaborativo mediante la herramienta informática Google Drive, en el desarrollo de la expresión escrita del idioma inglés

Vol. 2, núm. 4., (2018)

Ana María León Pazmiño; Andrea Patricia Albán Vinueza

Hay otro aspecto que también se debe tomar en cuenta, y es el hecho de tener varios lectores para un mismo párrafo; no solo será el profesor quien lo haga; sino que también los demás miembros del grupo realizarán la lectura del párrafo pertinente; en este proceso, los estudiantes podrán comentar, expresar sus diferentes puntos de vista respecto al tema objeto de la construcción del párrafo; logrando que estos sean auténticos.

Por lo expuesto, se propone investigar cómo mejoraría esta falencia si el trabajo se lo haría en línea de forma colaborativa, en donde inclusive las y los alumnas introvertidos estarían proactivamente participando por sentirse dentro de un entorno más seguro para él o ella, y donde contaría con la guía necesaria y la retroalimentación inmediata por parte del o la docente; así como también con el apoyo de sus compañeros o compañeras como correctores pares y colaboradores en la producción creativa de un párrafo.

En este marco, también se procedió a realizar una encuesta a los docentes del área de inglés donde se pudo verificar que nunca han trabajado con alguna plataforma digital para realizar trabajos colaborativos con sus estudiantes, y por ende, el $80 \%$ de ellos, no participan o aportan con sus ideas en las actividades propuestas por el docente. A pesar de que reconocen la importancia del trabajo en equipo no se han diseñado actividades enfocadas en el trabajo colaborativo a través de una plataforma virtual.

A partir de las dificultades encontradas en el pre-test y el desconocimiento del uso de aplicaciones digitales, como una metodología de enseñanza innovadora, por parte de los docentes, se elaboró una propuesta de actividades enfocadas en el trabajo colaborativo para 


\section{Incidencia del trabajo colaborativo mediante la herramienta informática Google Drive, en el desarrollo de la expresión escrita del idioma inglés}

Vol. 2, núm. 4., (2018)

Ana María León Pazmiño; Andrea Patricia Albán Vinueza

mejorar la producción escrita en las y los estudiantes universitarios mediante el uso de la herramienta informática Google Drive.

Los resultados obtenidos en el post-test, luego de la implementación de estas actividades evidenciaron un incremento significativo en su producción escrita, obteniendo una calificación de 7.34 sobre 10, es decir, se incrementó su desviación estándar en 65.7\% logrando desarrollar sus habilidades de la expresión escrita, cuyo producto final fue la construcción de un párrafo a través del trabajo colaborativo en el que participaron todos los estudiantes.

En este sentido, se pudo determinar la validez de la hipótesis alternativa donde se comprueba que la metodología propuesta sí influyó positivamente en el incremento de las calificaciones obtenidas y por tanto en su producción escrita.

Los resultados obtenidos del post-test permitieron plantear una propuesta sobre un Manual de Uso del Google Drive como herramienta digital para la implementación de estrategias del trabajo colaborativo en estudiantes del nivel básico del idioma inglés de la Universidad Central del Ecuador.

Los resultados de la investigación concluyeron que este proceso permitió identificar actividades adecuadas para mejorar la habilidad de la expresión escrita como la elaboración de párrafos en inglés como unidad básica de la comprensión de una lengua extranjera.

Para la escritura en inglés se deben tomar en cuenta estas consideraciones, ya sea dentro del lenguaje común como el técnico que exige una estructura distinta a las expresiones orales. Aquí la adecuación se define como la posibilidad de trabajar sobre las propias variantes del 
Incidencia del trabajo colaborativo mediante la herramienta informática Google Drive, en el desarrollo de la expresión escrita del idioma inglés

Vol. 2, núm. 4., (2018)

Ana María León Pazmiño; Andrea Patricia Albán Vinueza

idioma para poder utilizar el vocabulario adecuado. Es decir, para que un texto tenga sentido también debe responder al contexto a que se refiere; por lo que el docente debe saber guiar al estudiante en la composición de un texto, especialmente en construir ideas claras que puedan ser plasmadas en el papel; por lo que el papel del trabajo colaborativo mediante el uso de herramientas virtuales como Google Drive "se convierte en una estrategia que ayuda a reducir las dificultades lingüísticas en el proceso de adquisición del inglés, más aún, en el desarrollo de las habilidades de escritura” (Ubilla, Gómez, \& Sáez, 2017, pág. 345).

Esto debido a que en el contexto de la realidad de países de Latinoamérica como Ecuador ocurre que en el sistema educativo tanto de educación básica, media como superior existen muy pocas oportunidades de practicar de manera activa el inglés frenando así el desarrollo de habilidades no solo orales sino también de escritura.

\section{Trabajos citados}

Echazarreta, C., Prados, F., Poch , J., \& Soler, J. (2009). La competencia El trabajo colaborativo; una oportunidad para incorporar las TIC en la didáctica universitaria. Descripción de la experiencia con la plataforma ACME. Dossier Trabajo colaborativo, 1-11.

Gonnet, J. (2010). Reciprocidad, interacción y doble contigencia. Una aproximación a lo social. Revista de Filosofía A parte Reí, 1-10.

Hernández, R. (2010). Metodología de la Investigación. Mexico: McGrawHill.

Ibilib. (2013). Manual de uso avanzado de la aplicación Google Drive. La Mancha: Castilla: Junta de Comunidades de Castilla.

Maldonado, M. (2007). El trabajo colaborativo en el aula universitaria. Revista de Educación 13 (23), 263-278.

Ubilla, L., Gómez, L., \& Sáez, K. (2017). Escritura colaborativa de textos argumentativos en inglés usando Google Drive. Estudios pedagógicos, XLIII(1), 331-348. 